\title{
A study of Augmented Reality as a teaching and learning technology in the field of Design
}

\author{
Joyce Tavares \\ Technologies of Intelligence and Digital Design \\ Pontifícia Universidade Católica de São Paulo \\ São Paulo, SP, Brazil \\ jtavaresdesigner@gmail.com
}

\author{
Diogo Cortiz \\ Technologies of Intelligence and Digital Design \\ Pontifícia Universidade Católica de São Paulo \\ São Paulo, SP, Brazil \\ dcortiz@pucsp.br
}

\begin{abstract}
This paper aims to describe the results of a study about the effectiveness of Augmented Reality (AR) as a learning tool in Design. We proposed a design experiment to assess whether AR could improve learning in introductory drawing lessons. We used a sample of 60 students $(\mathrm{N}=60)$ from a technical school in the west of greater São Paulo for this purpose. The sample was divided into two groups: the control group (using just drawing regular tools) and the experimental group (using AR as an additional tool). Three expert reviewers were recruited to evaluate the final design of the participants. The reviewer evaluation results showed no statistical significance regarding reviewers' evaluation between control and experimental groups; however, a survey with the same students showed a statistical significance in their preference for using AR. A qualitative survey with participants also showed a preference for using AR. Based on those results, we argue that although the results did not show a significant difference from the evaluators' point of view, the students' perception suggests that AR can be an important auxiliary tool in the learning process.
\end{abstract}

\section{CCS CONCEPTS}

- CCS • Human-centered computing • Interaction design

\section{KEYWORDS}

Keywords: Augmented Reality, Education, Design

\section{ACM Reference format:}

\section{Introduction}

The article discusses the effectiveness of Augmented Reality (AR) as a learning tool in Design. Based on previous studies on the use of AR in education Ayer; Messner; Anumba[1]; Petrov and Atanasova[2] highlight its use in several areas. According to the New Jersey Institute of Technology [3], AR can be used even in the areas of Human Sciences, Natural Sciences, Technological Education and Arts.

Baldass et al. [4] proposed an experiment to measure whether AR could improve human performance in different tasks. They carried out a study with AR and Lego to analyze the efficiency of execution. They proposed the assembly of a certain piece with Legos in which one group used traditional instructions (paper) while the other group used instructions in AR. The group that used the AR instructions assembled the piece in a more efficient time and less fatigued brain, suggesting that the technology could help with other tasks. Inspired by this research, we proposed an experiment to measure how AR could improve learning processing in drawing classes. Next, we will outline a brief theoretical foundation and describe the methodology we used to apply our study and the results presented.

\subsection{Experimental protocol}

Our visual perception is related to our detection ability of light from the aesthetic and logical point of view, however we were not able to detect the incongruence parameter when we haven't even seen it, so making it difficult to identify the graphical flaw in the visual representation of a given object.

Our Research Question (RQ) is whether AR could improve learning in design area. The hypothesis is that augmented reality could help to expand the perception of space and images during learning experiences in courses involving graphic arts, often, students need to have visual examples to create their own representations and be able to have elements of their own authorship.

Starting from our research question, the main objective of the study is to assess where augmented reality can improve learning in design area. To answer our research question and achieve our objectives, the proposed study is a randomized controlled experiment, aiming to reduce the sources of bias by testing the effectiveness of new interventions. Thus, our study is a doubleblind, randomized, and controlled experiment, lasting one week, with a sample of 60 students $(\mathrm{N}=60)$. As inclusion criteria for the sample, we have the following parameters:

a) students from a public school system in the region of Carapicuíba who are starting their studies in Cultural Production and Design in courses technical level;

b) be over 16 years old. As an exclusion criterion for the sample, we have: a) not participating in the experience in full; b) not sending acceptance form;

c) Do not have some visual impairment that prevents visualization of the graphics.

Due to Coronavirus pandemic, this experiment was carried out remotely. The experimental group used an Augmented Reality filter developed by the authors, using the XYZ tool and available for smartphones. The control group did not use the AR filter, just regular tools. We apply Augmented Reality in a drawing training activity based on Andrew Loomis's [5] facial aesthetic proportion. 
The instructions given to the students were: in 10 minutes, draw a human face. The experimental group used the AR filter on mobile while the control group did not use any type of support.

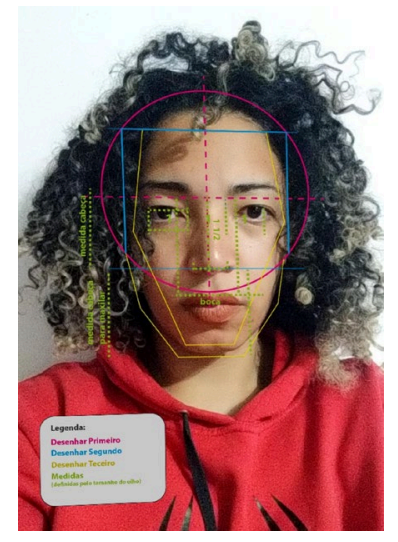

Figure 1: Figure Caption and Image above Instagram filter (copyright image).

Our hypothesis is that augmented reality will help to expand the perception of space and images during learning experiences, improving learning experience. In courses that involve graphic arts, students often need to have visual examples so that they can increase their repertoire and create their own representations to have elements of their own authorship.

To evaluate the students' results, we recruited three reviewers (teachers from the art and design area). In each round, each reviewer received two drawings, one produced by a participant in the experimental group and the other in the control group. Reviewers should use the most proportional Design as a choice criterion. If both were very similar, the tie would be for the best finished drawing (whichever looked more finished). We named the three reviewers $\mathrm{A}, \mathrm{B}$ and $\mathrm{C}$.

Both Reviewer A and Reviewer C chose $55.2 \%$ of the drawings by experimental group (using RA) while Reviewer B chose $48.3 \%$ of the drawings by experimental group. We performed $a=n$ One Sample Proportion Test (Binomial test) in the data. The results showed no statistical significance $(\mathrm{p}>0.05)$, as described in Table 1.

\begin{tabular}{llcrcc}
\multicolumn{1}{l}{ Binomial Test } & \multicolumn{1}{l}{ Level } & Count & Total & Proportion & $\mathrm{p}$ \\
\hline \multirow{2}{*}{ Grupo } & Controle & 41 & 87 & 0.471 & 0.668 \\
& Experimental (RA) & 46 & 87 & 0.529 & 0.668 \\
\hline
\end{tabular}

Note. $\mathrm{H}_{\mathrm{a}}$ is proportion $\neq 0.5$

\section{Table 1: Reviewers' evaluation (Binomial Test)}

A form was also applied to assess the students' perception of the use of Augmented Reality in the learning process. This survey was applied after each student had the opportunity to use Augment Reality. Of the total number of students participating in the experiment $(\mathrm{N}=60)$, a total of 52 students responded to our survey form. We had positive responses, with $73 \%$ of the students explicating their preference of using AR during the exercises. We performed a One Sample Proportion Test (Binomial test) in the data. The results showed statistical significance $(\mathrm{p}<0.05)$, as described in Table 2.

\begin{tabular}{llcrcc}
\hline \multicolumn{1}{l}{ Binomial Test } & \multicolumn{1}{c}{ Level } & Count & Total & Proportion & $\mathrm{p}$ \\
\hline \multirow{2}{*}{ Preference } & With RA & 38 & 52 & 0.731 & 0.001 \\
& Without RA & 14 & 52 & 0.269 & 0.001 \\
\hline
\end{tabular}

Note. $\mathrm{H}_{\mathrm{a}}$ is proportion $\neq 0.5$

Table 2: Student's preferences (Binomial Test)

\section{Conclusion}

The experimental results showed no statistical significance regarding reviewers' evaluation; however, a survey with students showed a statistical significance in their preference for using AR. A qualitative survey with participants also showed a preference for using AR. Based on those results, we argue that although the results did not show a significant difference from the evaluators' point of view, the students' perception demonstrates that $\mathrm{AR}$ can be an important auxiliary tool in the learning process.

We argue that those results open new possibilities to future research to study in each aspects $A R$ can improve learning experience, concentrating the research efforts to investigate students' perception and preferences of the technology. Those are some points that we aim to discuss in the dissertation.

Finally, it is important to note that the study was carried out during the pandemic, this may have affected the results, thinking that the test was applied remotely. A possible development is that it is interesting to apply the same test in person and not remotely to analyze the effectiveness of the method.

\section{REFERENCES}

[1] Ayer, Steven \& Messner, John \& Anumba, Chimay. (2016). Augmented Reality Gaming in Sustainable Design Education. Journal of Architectural Engineering. 22. 04015012. DOI:10.1061/(ASCE)AE.1943-5568.0000195

[2] Petrov, Plamen \& Atanasova, Tatiana. (2020). The Effect of Augmented Reality on Students' Learning Performance in Stem Education. Information (Switzerland). DOI:10.3390/info1 1040209

[3] New Jersey Institute of Technology's Online Masters in Computer Science program (2017) Why Augmented Reality Will Transform Education Infographic. http://graduatedegrees.online.njit.edu/resources $/ \mathrm{mscs} / \mathrm{mscs}$ infographics/augmented-reality-in-education/

[4] Baldassi, Stefano \& Cheng, Grace \& Chan, Jonathan \& Tian, Moqian \& Christie, Tim \& Short, Matthew. (2016). Exploring Immersive AR Instructions for Procedural Tasks: The Role of Depth, Motion, and Volumetric Representations. DOI:10.1109/ISMAR-Adjunct.2016.0101

[5] Andrew Loomis (1956). Drawing the Head and Hands ( $1^{\text {st }}$. ed.). Titan Books (UK) 